\title{
Lamotrigine effects on immune gene expression in larval zebrafish
}

\author{
Monika Mochol $^{\text {a,b,c, *, Paul Whatmore }}{ }^{\mathrm{d}}$, Erik Taubøll ${ }^{\mathrm{b}, \mathrm{c}}$, Cecilie Johannessen Landmark ${ }^{\mathrm{e}, \mathrm{f}, \mathrm{g}}$, \\ Erik Ropstad $^{\mathrm{h}}$, Sigrid Svalheim ${ }^{\mathrm{c}, 1}$, Thomas W.K. Fraser ${ }^{\mathrm{h}, 1,2}$ \\ ${ }^{a}$ Department of Neurology, Østfold Hospital Trust, P.O. Box 300, 1714 Grålum, Norway \\ ${ }^{\mathrm{b}}$ Institute of Clinical Medicine, University of Oslo, P.O. Box 1072 Blindern, 0316 Oslo, Norway \\ ${ }^{\mathrm{c}}$ ERGO - Epilepsy Research Group of Oslo, Department of Neurology, Oslo University Hospital, P.O. Box 4950 Nydalen, 0424 Oslo, Norway \\ ${ }^{\mathrm{d}}$ Research Office, DRI, Queensland University of Technology P Block, Gardens Point Campus, 2 George St, Brisbane, P.O. Box 2434, Brisbane, Australia \\ ${ }^{\text {e }}$ Program for Pharmacy, Faculty of Health Sciences, Oslo Metropolitan University, P.O. Box 4, 0130 Oslo, Norway \\ ${ }^{\mathrm{f}}$ The National Center for Epilepsy, Oslo University Hospital, P.O. Box 4950 Nydalen, 0424 Oslo, Norway \\ ${ }^{g}$ Section for Clinical Pharmacology, Dept of Pharmacology, Oslo University Hospital, P.O. Box 4950 Nydalen, 0424 Oslo, Norway \\ ${ }^{\mathrm{h}}$ Department of Production Animal Clinical Sciences, Faculty of Veterinary Medicine, Norwegian University of Life Sciences, P.O. Box 5003, 1432 Ås, Norway
}

\section{A R T I C L E I N F O}

\section{Keywords:}

Gene expression

Inflammation markers

Lamotrigine

Neuroinflammation

\begin{abstract}
A B S T R A C T
Purpose: Despite growing evidence that neuroinflammation and pro-inflammatory cytokines are involved in the pathogenesis of seizures and epilepsy, this knowledge has not been incorporated in the proposed mechanism of action of any of the current antiseizure medications (ASMs). Here, we tested the hypothesis by assessing inflammation markers in larval zebrafish (Danio rerio) exposed to lamotrigine (LTG).

Methods: In order to establish the most appropriate LTG concentrations for the transcriptome analysis (RNAseq), we initially assessed for teratogenic (spinal cord deformation, heart oedema, failed inflation of the swim bladder) and behavioural effects (distance moved, time spent active, and average swimming speed during a light/dark test) in zebrafish larvae exposed to $0,50,100,300,500,750$, and $1000 \mu \mathrm{M}$ LTG continuously between 5 and 120 $\mathrm{h}$ post fertilisation. Subsequently, we repeated the experiment with $0,50,100$, or $300 \mu \mathrm{M}$ LTG for transcriptomic analyses. Two databases (Kyoto Encyclopedia of Genes and Genomes; Gene Ontology) were used to interpret changes in gene expression between groups.

Results: Major teratogenic effects were observed at concentrations of $\geq 500 \mu \mathrm{M}$ LTG, whereas behavioural changes were observed at $\geq 300 \mu \mathrm{M}$ LTG. Transcriptome analysis revealed a non-linear response to LTG. From the suite of differentially expressed genes (DEG), 85\% ( $n=80$ DEGs) were upregulated following exposure to 50 $\mu \mathrm{M}$ LTG, whereas 58\% ( $n=12$ DEGs) and 91\% $(n=210$ DEGs) were downregulated in response to 100 and 300 $\mu \mathrm{M}$ LTG. The metabolic pathways affected following exposure to 50 and $300 \mu \mathrm{M}$ LTG were associated with responses to inflammation and pathogens as well development and regulation of the immune system in both groups. Notable genes within the lists of DEGs included component complement 3 (C3.a), which was significantly upregulated in response to $50 \mu \mathrm{M}$ LTG, whereas interleukin $1 \beta$ (IL-1 $\beta$ ) was significantly downregulated in the $300 \mu \mathrm{M}$ LTG group. The lowest exposure of $50 \mu \mathrm{M}$ LTG is regarded as clinically relevant to therapeutic exposure. Conclusion: We demonstrated that LTG had an impact on the immune system, with a non-monotonic response curve. This dose-dependent relation could indicate that LTG can affect inflammatory responses and also at clinically relevant concentration. Further studies are needed to establish this method as a tool for screening the effects of ASMs on the immune system.
\end{abstract}

Abbreviations: ASM, antiseizure medication; DEG, differentially expressed genes; DE, differential expression; DMSO, dimethyl sulfoxide; GO, Gene ontology; KEGG, Kyoto Encyclopedia of Genes and Genomes.

* Correspondence to: Department of Neurology, Østfold Hospital Trust, PO box 300, 1714 Grålum, Norway.

E-mail addresses: monikamochol@hotmail.com, monmoc@so-hf.no (M. Mochol).

1 Current working address: Reproduction and Developmental Biology, Institute of Marine Research (IMR), Matre Aquaculture Research Station, 5984 Matredal, Norway.

${ }^{2}$ Contributed equally 


\section{Introduction}

Over the past two decades neuroinflammation has been considered an important pathophysiological process involved in the development of epilepsy (van Vliet et al., 2018; Vezzani et al., 2019). The interleukin-1 receptor type 1 (IL-1R1) and toll-like receptor (TLR) pathways are frequently described as pivotal factors in seizure onset, recurrence, and epileptogenesis (Aronica and Crino, 2011; van Vliet et al., 2018; Vezzani and Granata, 2005). Interleukin-1 $\beta$ (IL-1 $\beta$ ), which is normally barely detected in healthy brains, is rapidly and persistently increased in the rodent hippocampus after induced status epilepticus (SE) (De Simoni et al., 2000). Immunohistochemical studies have also shown that IL-1 $\beta$ is produced in microglia and astrocytes during the chronic phase of spontaneous seizures in brain areas involved in seizure generation (Ravizza et al., 2008; van Vliet et al., 2018). Injection of IL-1 $\beta$ before kainic acid (KA) application in the rodent brains results in a pro-convulsant effect mediated by IL-1R1, whereas the intracerebral administration of the interleukin-1 receptor antagonist (IL-1Ra) leads to strong anticonvulsant effects (van Vliet et al., 2018; Vezzani et al., 1999, 2000). These findings have revealed an important role of IL- $1 \beta$ in ictogenesis. Furthermore, high mobility group box 1 (HMGB1), a protein that regulates gene transcription and activates TLR4, is rapidly and persistently induced in neurons and astrocytes in epilepsy (van Vliet et al., 2018). Studies on gene expression have also revealed activation of TLR4 and IL-1R1 signalling in human epilepsy (Aronica and Gorter, 2007).

Despite the growing evidence of neuroinflammation being an important contributor in epileptogenesis, little is known about whether this mechanism of action is regulated by current treatment options in epilepsy. Common pharmacological targets of antiseizure medications (ASM) are neuronal cells and ion channels (Brodie et al., 2011). For instance, lamotrigine (LTG) is frequently used to treat many types of epileptic seizures and for the treatment of other conditions such a bipolar disorder, migraine, and neuropathic pain, and to is known to inhibit neuronal voltage-gated sodium channels (VGSC) (Smith et al., 2007; Spina and Perugi, 2004). Further, in vitro studies revealed that LTG also reduces the level of IL- $1 \beta$ in the serum of healthy study participants (Himmerich et al., 2013). Uludag et al. found elevated serum levels of IL-1 $\beta$ in patients with temporal lobe epilepsy, although results were not correlated to the use of ASM (Uludag et al., 2015). However, in vivo concentrations of IL- $1 \beta$ in epilepsy patients using LTG have not been systematically investigated as research into the cytokine levels of ASMs in patients with epilepsy is challenging. There are some case reports and studies indicating that LTG affects the immune system in humans (Godhwani and Bahna, 2016; Svalheim et al., 2013). These findings suggest that our current knowledge of the mechanisms of action responsible for the broader range of therapeutic effects of ASM may be incomplete.

An improvement in our understanding of mechanisms involved in epileptogenesis could help create new therapies leading to seizures arrest, altering epilepsy progression, and preventing epilepsy in patients at risk. For this reason, animal models are an important prerequisite (Löscher, 2002). Rodent models have been widely used in epilepsy research, but have some obvious limitations for large-scale screening. The zebrafish (Danio rerio) has recently become an important model organism for the study of vertebrate development and pre-clinical screening in pharmaceutic research. The small size, ability to produce hundreds of offspring from a single spawning, transparent embryos and larvae, affordable maintenance, complex vertebrate system, including a similar genome (70-80\% homology with human genome (Dooley and Zon, 2000)) and brain structure as mammals, making them ideal for compound screening and translational studies (Barbazuk et al., 2000; Chakraborty et al., 2009; Gerlai, 2003; Howe et al., 2013). Taking advantages of these features and adapting electroencephalogram (EEG) recordings and registration of changes in gene expression related to epileptic seizure, has established zebrafish as an important model organism in epilepsy research (Baraban et al., 2005; Griffin et al., 2016; Hortopan et al., 2010; Stewart and Kalueff, 2012). As zebrafish larvae are legally considered an in vitro model prior to first feeding (i.e. 5 days post fertilisation), their use is encouraged within the $3 \mathrm{R}$ framework, to replace, reduce, and refine animal experiments. To this end, Baraban et al. revealed that chemically-induced seizures in zebrafish larvae demonstrated behavioural, electrographic, and molecular changes comparable to those in the rodent seizure model (Baraban et al., 2005). Therefore, although larvae have a reduced behavioural repertoire compared to adults, they are a viable model to study epilepsy which is aligned within the framework to reduce animal experimentation.

Due to knowledge gaps in the mode of action of current epilepsy treatments and the difficulties in studying the multiple mechanisms of action of ASMs, our aim was to evaluate the zebrafish model as a new alternative in studying the effects of ASMs on the immune system. To this end, we initially assessed the teratogenic and behavioural response to zebrafish larvae exposed to a large range of LTG concentrations, one of the most commonly used ASMs. Following this, we used three nonlethal/teratogenic concentrations for transcriptome analysis to test our hypothesis that LTG will have an effect on the immune system.

\section{Materials and methods}

\subsection{Chemicals}

Dimethyl sulfoxide (DMSO) (purity, $>99.7 \%$, CAS number 67-68-5) and LTG (purity, $\geq 98 \%$, CAS number 84057-84-1) were purchased from Sigma-Aldrich. All stock solutions of lamotrigine were prepared in DMSO on the day of testing.

\subsection{Fish husbandry}

The study was conducted at The Norwegian University of Life Sciences (NMBU), Oslo, Norway. The research was performed according to the regulations approved by the unit's animal ethics committee (Institutional Animal Care and Use Committee/IACUC) following Norwegian laws and regulations controlling experiments and procedures on live animals in Norway. AB wild-type zebrafish were maintained at $28 \pm 1{ }^{\circ} \mathrm{C}$ under a 14:10 light/-dark photoperiod. Animal care was carried out in accordance with the local protocols. To generate embryos, adults were placed in spawning tanks in the afternoon and spawning took place the next morning when the lights were turned on (08:00). The embryos were collected after one hour and maintained in sterile embryo media $(60 \mu \mathrm{g} /$ $\mathrm{mL}$ Instant Ocean ${ }^{\circledR}$ sea salts) until the time of exposure.

\subsection{Preparation of solutions}

For behavioural experiments, six concentrations of LTG in 1\% DMSO were used. The concentrations of LTG were chosen based on previous studies investigating the efficacy as well neurotoxic, sedative and teratogenic potential of ASM (Berghmans et al., 2007; Lee et al., 2013). LTG solutions were prepared by diluting the stock solution of $100 \mathrm{mM}$ LTG in sterile embryo media to $1000 \mu \mathrm{M}$ LTG in $1 \%$ DMSO, and further diluting this stock solution with $1 \%$ DMSO to $750 \mu \mathrm{M}, 500 \mu \mathrm{M}, 300 \mu \mathrm{M}, 100 \mu \mathrm{M}$, and $50 \mu \mathrm{M}$ LTG (exposers will be referred to as LTG 1000, LTG 750, LTG 500, LTG 300, LTG 100, LTG 50). Originally, 1\% DMSO was used to ensure the highest concentration of LTG $(1000 \mu \mathrm{M})$ was in solution, which could not be achieved with $0.1 \%$ DMSO. For the transcriptome analysis, we prepared a stock solution of $300 \mathrm{mM} \mathrm{LTG}$ in $0.1 \%$ DMSO which was further diluted with $0.1 \%$ DMSO to LTG 300, LTG 100, LTG 50 . We chose to use $0.1 \%$ DMSO for RNA-sequencing and transcriptome analysis as it was sufficient to ensure $300 \mathrm{mM} \mathrm{LTG}$ was in solution, it is commonly used in zebrafish studies (Parsons et al., 2019), and it is known to not effect larval behaviour (Christou et al., 2020). To be sure that we achieved the desired concentrations, the concentration of stock solution was determined. 
The analytical procedure for the analysis of LTG concentrations were handled as routine measurements using validated methods for serum concentration measurements at the Section for Clinical Pharmacology, The National Center for Epilepsy, Oslo University Hospital. The analytical method was high-performance liquid chromatography with ultraviolet (UV) detection on an Agilent 1200 HPLC system 3. The column was a C18-synergi 4 u Hydro-RP 80 A $250 \times 3.0 \mathrm{~mm}$. The mobile phase consisted of methanol/acetonitrile, and citalopram was used as internal standard. Limits of quantification were $2.0-75 \mu \mathrm{mol} / \mathrm{L}$ with an analytical coefficient of variation (CV) $<10 \%$. The method underwent regular quality control including internal controls and external LGC standards for evaluation. The method was based on Contin et al. (2010).

\subsection{Exposures for behavioural tests}

Fertilized embryos were transferred into clear polystyrene 96-well plates (Nunc ${ }^{\mathrm{TM}}$ MicroWell ${ }^{\mathrm{TM}}$ ) with one embryo per well and continuously exposed under static conditions from $5 \mathrm{~h}$ post fertilisation (hpf) until the time of testing at $116-120 \mathrm{hpf}$ in $300 \mu \mathrm{L}$ of media. Embryos were exposed to one of six LTG concentrations (LTG 1000, LTG 750, LTG 500, LTG 300, LTG 100, LTG 50) or the control (1\% DMSO). All groups were spread equally on each row and column to avoid position-bias during testing. Each well plate included 12 embryos per media and was repeated in four independent experiments.

\subsection{Exposures for transcriptome analysis}

Based on behavioural tests we choose three concentrations of LTG (LTG 50, LTG 100, LTG 300) to work with during transcriptome analysis. Fertilized embryos were placed in six well plates (Falcon ${ }^{\circledR} 6$ 6-well) with either one concentration of LTG or the control ( $0.1 \%$ DMSO). In each well, 15 embryos were immersed in $3 \mathrm{~mL}$ of media and exposed from 5 hpf until $120 \mathrm{hpf}$. Six replicates of 12 non-deformed embryos were collected, snap-frozen in liquid nitrogen, and stored at $-80^{\circ} \mathrm{C}$ until further analysis.

\subsection{Behavioural test}

Analysis were performed in a ViewPoint ${ }^{\circledR}$ Zebrabox and its tracking software (ViewPoint Life Sciences, Lyon, France). The Zebrabox consists of an arena in which light, temperature, and sound can all be manipulated, and digital video captured, so as to analyse the movement of up to 96 larvae simultaneously. It is frequently used in toxicology/pharmacology research (Christou et al., 2020). Behavioural assays consisted of maintaining larvae in a light dark cycle (10 min light followed by $10 \mathrm{~min}$ in dark) after a $10 \mathrm{~min}$ acclimation period in the light as commonly used in our lab (Christou et al., 2020) and others (Orellana-Paucar et al., 2012). All tests were registered between 9:00-13:00 in 116-120 hpf zebrafish larvae. The cumulative distance moved and the time spent active were simultaneously measured in all larvae on a given well-plate. Subsequently, swimming speed was calculated by dividing the distance moved by the time spent active.

\subsection{RNA extraction and purification}

Each of the 16 samples (containing 12 pooled larvae) were completely homogenized by using 21-gauge needles (HSW HENKEJECT ${ }^{\circledR}$, Germany). RNA was purified using the NucleoSpin ${ }^{\circledR}$ RNA extraction kit (Macherey-Nagel, Germany). Total RNA was extracted from samples following the manufacturer's instructions. Each sample was eluted in $50 \mu \mathrm{L}$ RNase-free water and stored at $-80^{\circ} \mathrm{C}$ until further analysis. A Nanodrop-1000 Spectrophotometer (NanoDrop Technologies, DE, USA) was used to assess RNA purity. RNA integrity number (RIN) was determined using an Agilent 2100 Bioanalyzer, with a RNA Nano LabChip Kit (Agilent Technologies, Ca, USA). All samples had acceptable quality (RIN scores $>9.0$ ) and were subsequently utilized for further sequencing analysis.

\subsection{Transcriptome sequencing}

Extracted RNA was sent to Novagene Co., Ltd for sequencing. Libraries were prepared using a custom pipeline including the following steps: RNA purity was initially tested using a Nanodrop 8000 (Thermo Scientific, Wilmington, USA) and samples were run on Agarose Gel Electrophoresis to test for degradation and contamination, whereupon a final purity test was completed on an Agilent 2100 Bioanalyzer. Samples that passed these quality control steps underwent mRNA enrichment using oligo(dT) beads. The enriched mRNA was randomly fragmented using fragmentation buffer and then cDNA first-strand was synthesised by reverse transcriptase with random hexamers. Second-strand synthesis was by Escherichia coli polymerase I nick-translation with dNTPs and RNase $\mathrm{H}$. The final cDNA library was completed using terminal repair, adapter ligation, size selection, PCR enrichment and A-tailing. Sample libraries underwent a final quality control check before being sequenced on an Illumina NovaSeq 6000, producing 150 base pair, paired-end reads.

\subsection{Sequence quality control, genome alignment and gene expression quantification}

Quality control of the sequences was completed at Novagene Co., Ltd, using the Bcl2fastq 2.0 (Illumina) tool, which included demultiplexing, conversion from BCL to fastq format, adapter removal and quality filtration of reads $<\mathrm{Q} 30$ and 1 or more base mismatches. When the sequences were downloaded from Novogene, a confirmatory assessment of sequence quality and adapter contamination was completed using Fastqc v0.11.8 (Andrews, 2010).

Sequences were aligned to the zebrafish NCBI reference genome (ftp://ftp.ncbi.nlm.nih.gov/genomes/all/) using HISAT2 v2.1.0 (Kim et al., 2015) with default parameters. Gene expression was quantified using feature Counts v1.6.0 (Liao et al., 2014), being the number of sequences that aligned to exons within a gene region, where exon and gene regions was defined by the GRCz11 General Feature Format (GFF) annotation file.

Quality filtered sequence files (fastq format) were uploaded to Sequence Read Archive (https://www.ncbi.nlm.nih.gov/sra). The project accession number is BioProject ID PRJNA704066.

\subsubsection{Differential gene expression and metabolic pathway analysis}

All downstream analysis was completed in $\mathrm{R}$ version 3.6 .2 ( $\mathrm{R}$ Development Core Team, (http://cran.rproject.org). Prior to differential expression (DE) analysis, samples were examined for batch effects and outliers using base R tools to generate density plots, PCA plots, pairwise sample comparisons and hierarchical clustering. For DE analysis the DESeq2 package (Love et al., 2014) was used to compare each of the three LTG treatment groups (LTG 50-300) to the controls. The control groups were the baseline group in all comparisons, therefore genes with positive log fold change represent upregulation following LTG exposure and negative log fold change representing downregulation. For each gene, DESeq2 estimates fold change and expression strength between experimental treatments using a negative binomial generalized linear model. Significant differences in gene expression between treatments are estimated by a Wald test and the $p$ values are then adjusted for false discovery rates (FDR) using Benjamini-Hochberg (Benjamini and Hochberg, 1995). We considered genes to be significantly differentially expressed if they had an adjusted p-value of $<0.05$ and a $\log 2$ fold change of \pm 1 .

The list of DE genes was used to assess over or under-representation in Kyoto Encyclopedia of Genes and Genomes (KEGG) pathways and Gene Ontology (GO) terms. The ClusterProfiler package v3.6.0 (Yu et al., 2012) was used to perform this enrichment analysis, which performs a hypergeometric test to estimate overrepresentation of differentially 
expressed genes (DEG) per pathway. Significantly enriched pathways were those with FDR adjusted (again, using Benjamini-Hochberg) p-values of less than 0.05 .

Additional assessment of functional molecular categories, including protein domains, was completed using the Database for Annotation, Visualization and Integrated Discovery (DAVID, http://david.abcc.ncifc rf.gov). Protein domain databases examined by DAVID included INTERPRO (https://www.ebi.ac.uk/interpro/), PFAM (http://pfam.xfa m.org) and SMART (http://smart.embl-heidelberg.de/). Enrichment of individual categories was quantified using a modified Fisher Exact pvalue, generating an Expression Analysis Systematic Explorer (EASE) score, based on the recommended a significance threshold of 0.1 (Huang da et al., 2009). Functional relationships between categories were estimated using a Kappa statistics score that measured the co-association of DE genes between annotation groups, which were then clustered using fuzzy heuristic clustering, generating an enrichment score (Huang et al., 2007). Significantly clustered groups were based on an enrichment score of $>2$.

\subsection{Analysis of behavioural data}

Behavioural data was analysed in R version 3.2.3 (R Development Core Team, http://cran.rproject.org/). All dead and deformed larvae were discounted for behavioural analyses. For all test compounds, only motility during the dark phase was analysed as movement was minimal during the light periods. We used linear mixed effect (LME) models within the "nlme" package of $\mathrm{R}$ to assess behaviour. The dependent variable was either the cumulative time spent active (seconds), the cumulative distance travelled ( $\mathrm{mm}$ ), or average swimming speed (calculated as the cumulated distance travelled/cumulated time spent active), with concentration as a categorical independent variable, and replicate as a random effect. Only those larvae that moved more than one body length $(4 \mathrm{~mm})$ during the test period were included in the analysis. The lsmeans package was used for post-hoc analyses for significant main model effects. For all models, examination of the residual plots verified that no systematic patterns occurred in the errors (e.g. q-q plots). Significance was assigned at $p=<0.05$.

\section{Results}

\subsection{Teratogenic and behavioural result of larvae exposure to LTG and compound analysis}

All larvae exposed to $1000 \mu \mathrm{M}$ LTG died. The same results were observed in 3 of the 4 replicates for larvae immersed in $750 \mu \mathrm{M}$ LTG. Larvae exposed to $500 \mu \mathrm{M}$ LTG had varying prevalence of deformation (6,4\% spinal cord deformation, 3,2\% heart oedema, 9.7\% swimming bladder) and mortality (16,7-100\%). There was no significant difference in mortality or the prevalence of deformation at $\leq 300 \mu \mathrm{M}$ compared to controls (Fig. 1).

There were no significant behavioural differences between controls and LTG 50 and LTG 100, whereas LTG 300 and LTG 500 resulted in a significant decrease in the total time spent active and the total distance moved compared to controls (Fig. 2).

Compound analysis showed accordance between the calculated LTG concentrations and the final exposure concentrations measured in the solutions. For the calculated concentrations used in transcriptome analysis 50, 100, and 300, the corresponding mean concentrations in the medium were 56.4, 114.2, and 336.6 $\mu \mathrm{M}$ (with Range [53.1-60] $\mu \mathrm{M}$, [105.5-119.0] $\mu \mathrm{M},[317.3-359.0] \mu \mathrm{M})$.

\subsection{Sequencing analysis}

FastQC analysis showed high quality of sequences with Phred score between 34 and 36.5 over all reads. The total number of reads per sample varied from 20.5 million to 27.4 million, with an average of 24.7

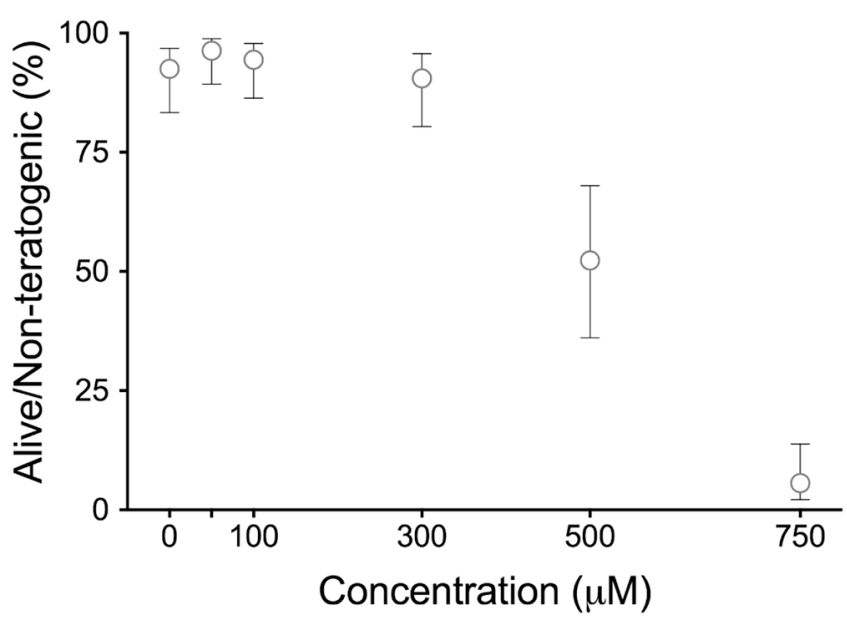

Fig. 1. Results of teratogenic effect due to exposure to different lamotrigine concentrations. The horizontal axis displays lamotrigine concentration, while the vertical axis represents the percentage of healthy zebrafish larvae. Results are based on 4 independent exposers with 12 zebrafish larvae in each tested compound.

million reads. Most reads aligned to the zebrafish reference genome, with an average alignment rate of $89.4 \%$ for all samples. Of the reads that aligned to the reference genome, about $55.4 \%$ were assigned to defined gene regions, based on RefSeq genome annotation definitions.

\subsubsection{RNA seq expression results and pathways analysis}

DESeq2 analysis revealed 80 significantly DEG for LTG 50 (12 downregulated, 15\%), 12 DEG for LTG 100 (7 downregulated, 58\%), and 210 for LTG 300 (191 downregulated, 91\%) (Fig. 3).

KEGG analysis revealed several significantly enriched pathways in LTG 300, primarily receptor signalling pathways (C-type lectin receptor signalling pathway, NOD-like receptor signalling pathway, Toll-like receptor signalling pathway and RIG-I-like receptor signalling pathway). IL-1 $\beta$ was downregulated in three of those pathways (Fig.A1:A3). GO analysis for biological function detected numerous processes associated with response to pathogens, the inflammatory response, as well development and regulation of immune system (Fig. 4). For LTG 100, KEGG results revealed 3 significantly enriched pathways. None of those pathways were directly associated with the immune response. Further, the gene count involved was low. KEGG analysis in LTG 50 showed significantly enriched pathways involved in neuroactive receptor-ligand interactions. In this treatment, complement component 3a (C3.a) was significantly upregulated. Studies of GO terms revealed multiple pathways associated with regulation of the immune response (Fig. 4).

DAVID analysis identified 44 enriched protein domains in LTG50 (Table.A4 in appendices), 4 domains in LTG300 (Table.A6 in appendices) and 0 domains in LTG50. These clustered into 4 significantly enriched clusters for LTG50 (Table.A5 in appendices) and 2 clusters for LTG300 (Table.A7 in appendices). For LTG50 the most significant protein domains, and the most highly enriched cluster (enrichment score 8.43) were dominated by alpha 2 macroglobulins (A2M). Also significantly enriched domains and clusters for LTG50 were peptidases/trypsins (effect size (e.s.) 7.84), serine protease inhibitor (e.s. 3.86) and fibrinogens (e.s. 2.01). The 2 enriched LTG300 clusters were nidogen (e. s. 3.22) and basic leucine zipper domains (e.s. 2.83).

\section{Discussion}

The main aim of the study was to assess the effect of LTG on the gene expression of inflammation markers in the larval zebrafish model. We found some support for our hypothesis that LTG effects the immune system, as we found a general pattern of upregulation of inflammation 
(A)

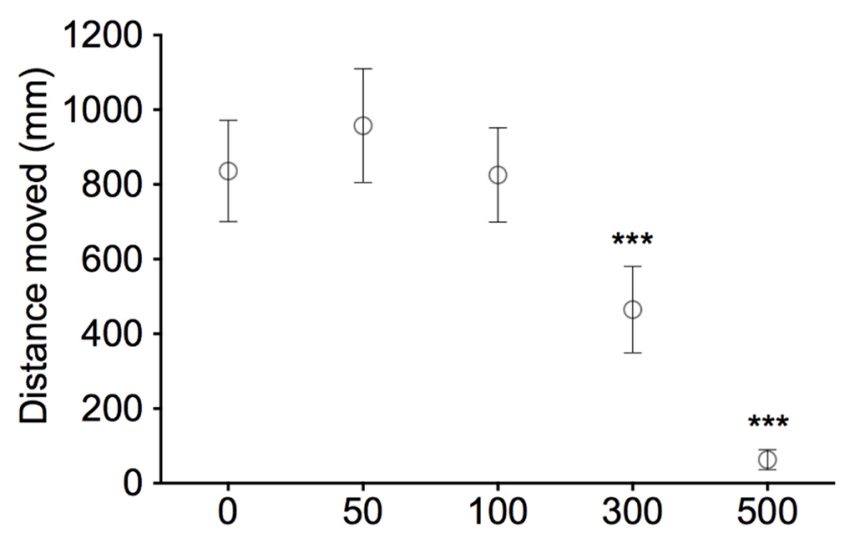

(B)

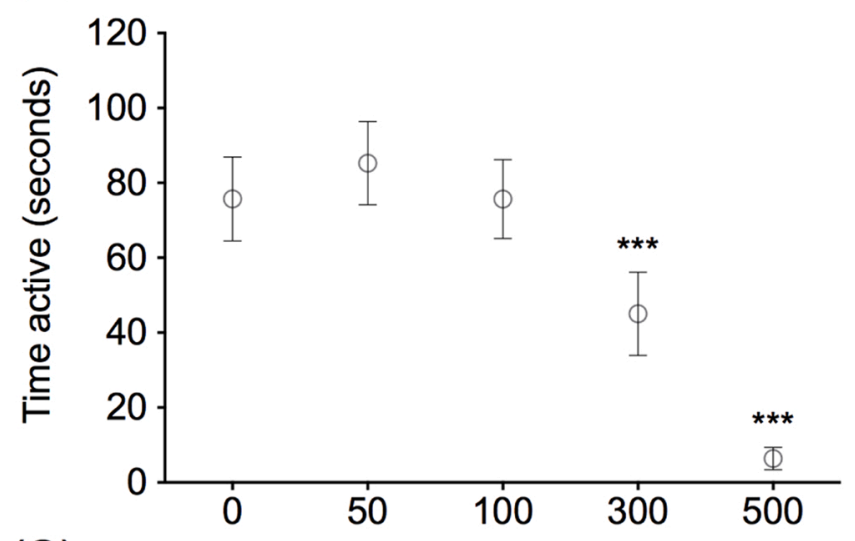

(C)

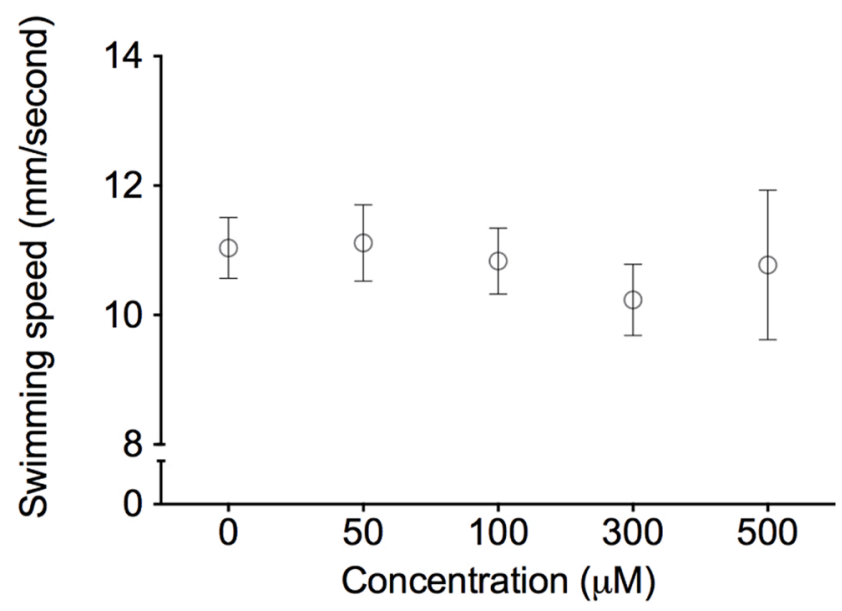

Fig. 2. Larval behaviour following exposure to lamotrigine. (A) Cumulative distance moved, (B) cumulative time spent active, and (C) the mean swimming speed. Results based on 4 independent exposures with 12 zebrafish larvae in each of the tested compound. Data are means \pm the $95 \%$ confidence interval. The statistics are from linear mixed effect models. An asterisk $(* * *)$ indicates a significant effect compared to the control (least square means, $p<0.001$ ).

markers in zebrafish exposed to LTG 50, whereas downregulation of inflammation markers occurred for LTG 300. Our results are discussed in relation to possible novel mechanisms of action for LTG.

The main finding in our study is that LTG influences gene expression of inflammation markers in zebrafish larvae. However, the response is not linear as $85 \%$ of the DEG were upregulated following treatment with
LTG 50, whereas $91 \%$ were downregulated with LTG 300. This type of response can be described as a non-monotonic dose response, whereby the response curve has a change in sign (i.e. from positive to negative or vice versa) over the range of doses tested (Conolly and Lutz, 2004; Kohn and Melnick, 2002). This dose-related response was more prominent when investigating differences within the LTG group. Fig. 5 shows lower gene expression of component complement 3 (C3) group with increased LTG concentration. However, the results for LTG 300 were not significantly different from the control group. Non-monotonic dose responses may explain why an increasing dose of some ASMs can lead to the exacerbation of seizures. This effect was also described in patients using LTG (Bauer, 1996; Guerrini et al., 1998). The possible mechanisms include an increased vulnerability to other medications or neurotoxins, an altered receptor-response to drugs, or possibly an incipient toxic effect at the highest dose (Bauer, 1996; Conolly and Lutz, 2004).

Component complement 3 (C3) was upregulated in the majority of the GO biological function terms assigned to diverse immunological processes in LTG 50. C3.a is a protein generated from complement pathway activation and has an important function in pathogenic infection. It affects T cell activation and survival (Strainic et al., 2008) as well as stimulating chemotaxis and macrophage activation and has a dichotomous action (Mathern and Heeger, 2015). During the acute phase of inflammation, it has an anti-inflammatory effect, in contrast, in chronic inflammation it acts as a proinflammatory molecule. C3.a may also enhance or decrease production of cytokines from peripheral blood mononuclear cells, among them IL-1 $\beta$ (Coulthard and Woodruff, 2015), and is reported to be one of the inflammatory compounds which alter blood-brain barrier permeability (Oby and Janigro, 2006). C3 has previously been linked to epilepsy, with it being significantly higher in untreated patients compared to heathy controls (Başaran et al., 1994), whereas others have found an increased expression of complements, among them C3, after SE in rodents and humans with temporal lobe epilepsy (Aronica et al., 2007; Schartz et al., 2018). These data indicate that complement activation could contribute to a sustained inflammatory response, which could imply that LTG 50 by increasing expression of C3.a could have an impact on epileptogenesis. The majority of DEGs from the LTG 300 were downregulated. All the significantly enriched KEGG pathways are involved in immunological responses to pathogens or stimulate inflammation (Chen et al., 2016; Geijtenbeek and Gringhuis, 2009). Of particular interest is the impact of LTG 300 on the TLR-signalling pathway. Previous studies have shown the expression level of TLR4 mRNA is positively correlated with the number of seizures in patients with epilepsy (Aronica and Gorter, 2007; Pernhorst et al., 2013). An in vitro study on blood from healthy patients reported a reduced level of IL-1 $\beta$ after treatment with LTG (Himmerich et al., 2013). The TLR- and IL-1R-signalling pathways are both activated by IL-1 $\beta$ and are central in epileptogenesis. Experimental studies using anti-inflammatory molecules acting as antagonists to IL-1R and the TLR pathway observed a 70-90\% reduction in the frequency of spontaneous seizures in chronic epilepsy (Iori et al., 2017). Based on our results, we could hypothesize that the downregulation of the IL-1 $\beta$ and TLR-signalling pathway by LTG 300 could have an anti-inflammatory effect and consequently have an impact on epileptogenesis. Further studies could then determine whether LTG does result in reduced protein levels of IL-1 $\beta$ in zebrafish. If so, one could then assess whether treatment with a high-dose of LTG could be preferred in patients at risk of developing epilepsy after a stroke or brain injury.

Protein domain analysis revealed significantly enriched A2M in LTG50. This protein is a major component of the innate immune system that increases during the early stages of inflammation. A2M has been associated with Alzheimer disease, febrile seizures, and acute disseminated encephalomyelitis (Suzuki et al., 2019; Varma et al., 2017). However, A2Ms involvement in epilepsy has not been previously described. Other enriched protein domain clusters provide further support for LTGs role in inflammation. Peptidase S1 (encoded by cathepsin $\mathrm{G}$ ) plays a role in remodelling connective tissue at inflammation sites in 


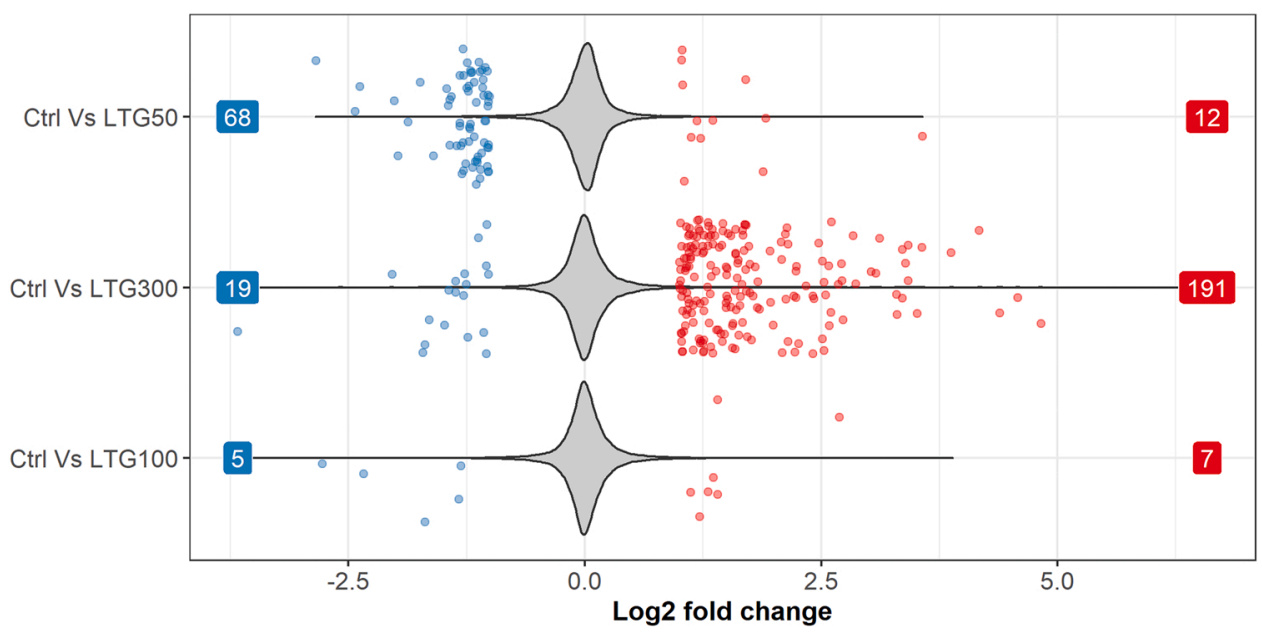

Fig. 3. Proportion of upregulated and downregulated genes. Log2 fold change scores of significantly different expressed (DE) genes are represented by jittered dots (blue $=$ significantly upregulated, red = significantly downregulated). Violin plot represents non-significant log2 fold change scores. Ctrl, control; LTG 50, lamotrigine $50 \mu \mathrm{M}$; LTG 100, lamotrigine $100 \mu \mathrm{M}$; LTG 300, lamotrigine $300 \mu \mathrm{M}$.

(A)

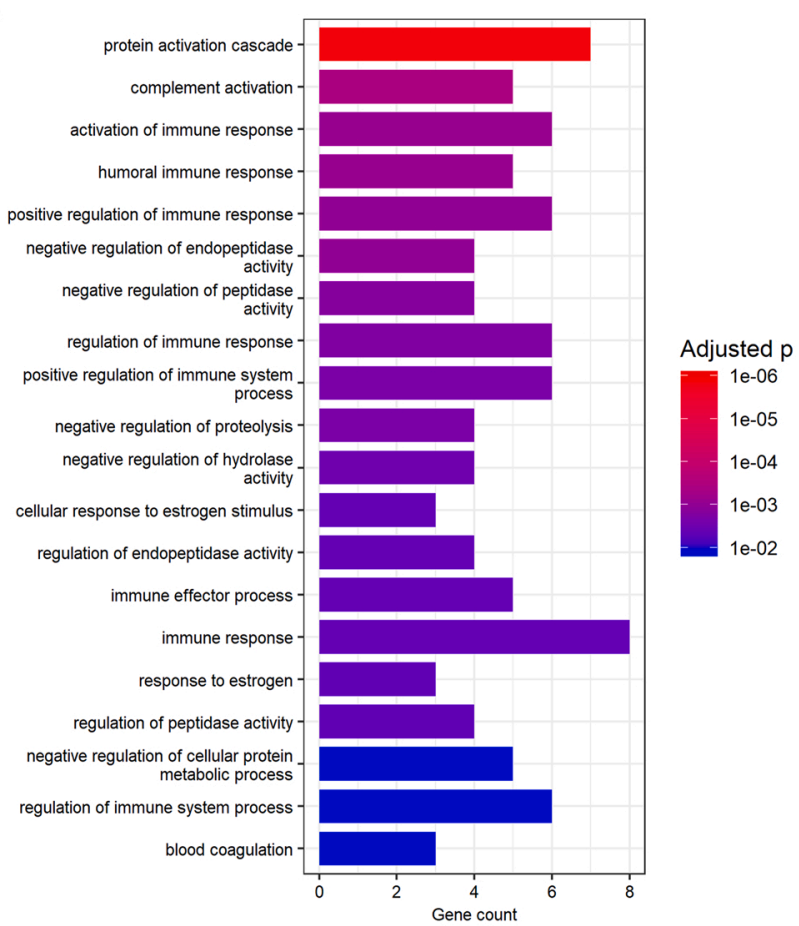

(B)

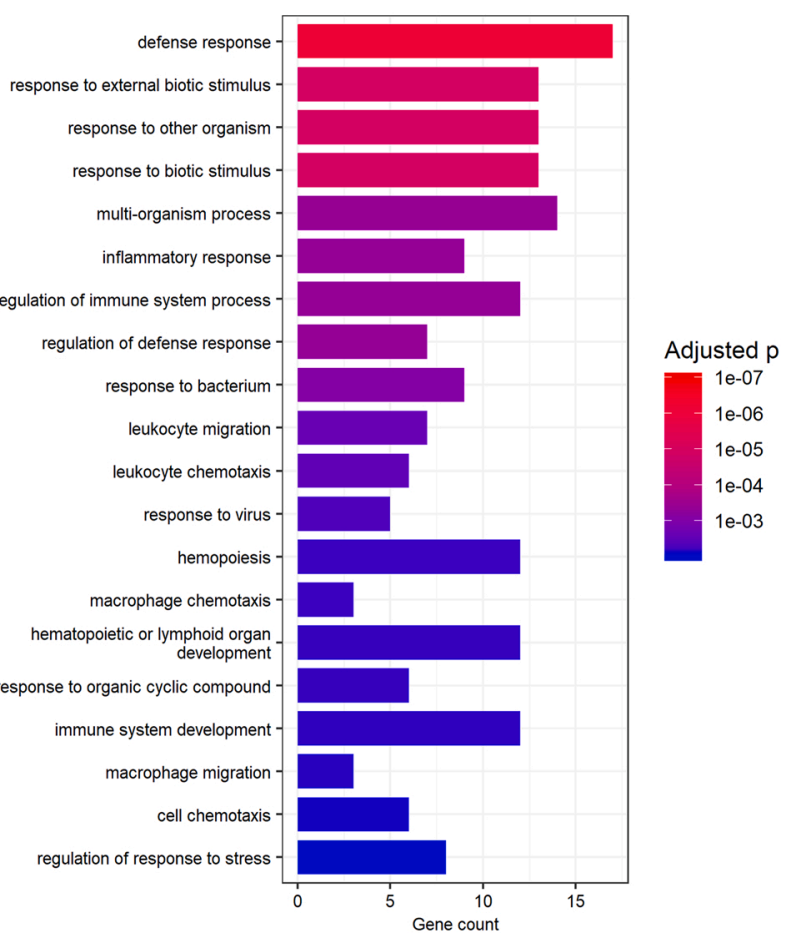

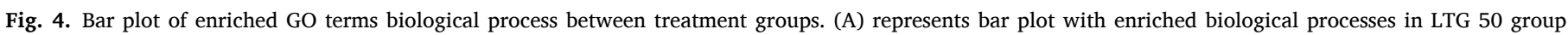

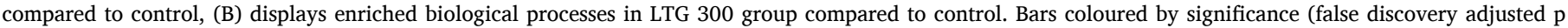

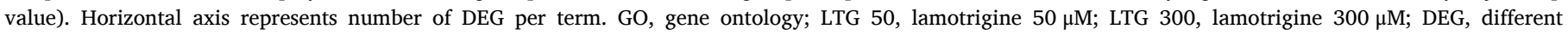
expressed genes.

myeloid leukaemia patients (Alatrash et al., 2017). Serine protease inhibitors (SERPIN) are involved in wide range of processes - coagulation, inflammation, digestion and are also present in CNS. The aberrant activity of these molecules has been described in Alzheimer's disease, Parkinson's disease, traumatic brain injury, and stroke (Almonte and Sweatt, 2011). SERPIN have been causatively linked to epilepsy (Roussel et al., 2016). The majority of (above-described) protein domain enrichment occurred at LTG50, suggesting that an inflammation response occurs also at therapeutic concentrations of LTG.

The mechanistic actions by which LTG regulates the immune system are unknown. LTG likely acts by inhibiting sodium currents by selectively binding to inactive sodium channels. Other VGSC blocking ASMs like phenytoin and carbamazepine are well known to modulate immunoglobulin levels (Yamamoto et al., 2010). In line with that, clinical studies have shown that LTG induce hypogammaglobulinemia (Svalheim et al., 2013) and causes adverse uncontrolled immune reaction hemophagocytic lymphohistiocytosis (Kim et al., 2019). Previously, it has been reported that sodium channels play a role in the release of inflammatory cytokines from microglia stimulated with LPS (Hossain et al., 2013). It has also been demonstrated that treatment with the VGSC blockers phenytoin and carbamazepine ameliorates the inflammatory response and has protective effects on central nervous system axons, thus may act as a neuroprotectant in experimental autoimmune encephalitis, a model of MS (Black et al., 2007; Black and Waxman, 


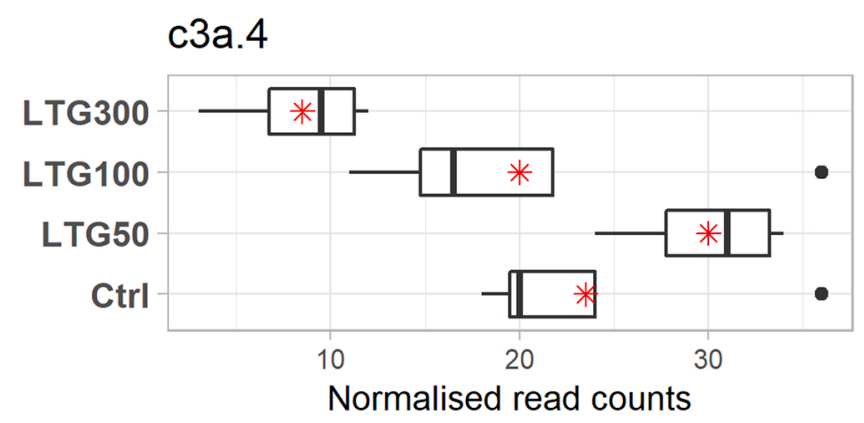

Fig. 5. The figure presents box plot illustrating negative trend: expression levels decrease with increased dose. As example-the expression levels of C.3a4 genes decrease with increased LTG concentration. Red stars-present means of read counts; back dots-presents outliers.

2008; Craner et al., 2005). It is therefore tempting to suggest that LTG may have acted on immune gene expression via its role as an VGSC blocker, although more studies have to be done to support this assumption.

The concentrations of LTG used for the transcriptome analysis were based on results from our preliminary toxicology and behavioural experiment. The lowest exposure is regarded as comparable to exposure of LTG during therapeutic exposure. Similar to other studies, we did not find any indications of a teratogenic effect in larvae expose to LTG at concentrations of $\leq 300 \mu \mathrm{M}$. Furthermore, LTG was found to increase the prevalence of deformities and mortalities at $\geq 500 \mu \mathrm{M}$ as previously observed (Berghmans et al., 2007; Lee et al., 2013). However, LTG 300 had a significant effect on basal locomotor activity that was unexpected, since it was previously reported to have no sedative effect at 30-300 $\mu \mathrm{M}$ in a similar behavioural test in larval zebrafish (Berghmans et al., 2007). Nevertheless, although not significant, there was a trend for reduced movement in larvae exposed to increasing concentrations of LTG in the study of Berghmans et al. (Berghmans et al., 2007). As the LTG 300 group was essentially the highest dose that did not cause a teratogenic effect, it is unclear whether this behavioural effect is due to general toxicity or a specific mode of action related to LTG. However, the immune system is known to play a significant role in behaviour and a large-scale screen in larval zebrafish concluded that immune-suppressants reduce activity during light/dark tests due to their role in setting normal activity levels (Rihel et al., 2010). As the LTG 300 treatment led to downregulation of immune pathways, this treatment could be considered to have an immune suppressive effect that may then explain the reduction in general activity.

Several study limitations are acknowledged. In the current study we used four biological replicates per LTG treatment group, which was sufficient to detect large expression differences between treatments for the individual genes. However, a larger number of biological replicates would have enabled a finer resolution of DEGs, resulting in more detected DEGs and subsequently an improved identification of significantly enriched KEGG pathways and GO terms (due to enrichment being based on the proportion of detected DE genes to the total number of genes in a pathway/term). It is also important to mention that not all genes are expressed at the early stage of development. Finally, it should be noted that there is still a gap between preclinical findings and possible clinical implications when it comes to treatment of patient, which should be cautiously interpreted.

\section{Conclusion}

Our study aimed to determine whether LTG impacts on the immune system in a vertebrate model used extensively in biomedical research. We have demonstrated that LTG has an impact on the immune system following sub-lethal exposure, with a non-monotonic dose response curve. LTG could affect inflammatory responses and reduce epileptogenesis also at clinically relevant concentrations. Further studies should focus on investigating changes in genes expression and levels of inflammatory markers in zebrafish treated with LTG and other ASMs before and after epileptic seizures to help close the knowledge gap between preclinical findings and possible clinical implications in patients.

\section{Funding}

This work was supported by Norwegian Epilepsy Federation (Norsk Epilespiforbund) and Norwegian Epilepsy Society (Norsk Epilepsiselskap).

\section{Declaration of interest}

None.

\section{Appendix A. Supporting information}

Supplementary data associated with this article can be found in the online version at doi:10.1016/j.eplepsyres.2021.106823.

\section{References}

Alatrash, G., Garber, H.R., Zhang, M., Sukhumalchandra, P., Qiu, Y., Jakher, H., Perakis, A.A., Becker, L., Yoo, S.Y., Dwyer, K.C., Coombes, K., Talukder, A.H., John, L.S.S., Senyukov, V., Lee, D.A., Sergeeva, A., He, H., Ma, Q., Armistead, P.M., Roszik, J., Mittendorf, E.A., Molldrem, J.J., Hawke, D., Lizee, G., Kornblau, S.M., 2017. Cathepsin G is broadly expressed in acute myeloid leukemia and is an effective immunotherapeutic target. Leukemia 31, 234-237.

Almonte, A.G., Sweatt, J.D., 2011. Serine proteases, serine protease inhibitors, and protease-activated receptors: roles in synaptic function and behavior. Brain Res. 1407, 107-122.

Andrews, S., 2010 FastQC: a quality control tool for high throughput sequence data.

Aronica, E., Crino, P.B., 2011. Inflammation in epilepsy: clinical observations. Epilepsia 52 (Suppl 3), 26-32.

Aronica, E., Gorter, J.A., 2007. Gene expression profile in temporal lobe epilepsy. Neuroscientist 13, 100-108.

Aronica, E., Boer, K., van Vliet, E.A., Redeker, S., Baayen, J.C., Spliet, W.G.M., van Rijen, P.C., Troost, D., Lopes da Silva, F.H., Wadman, W.J., Gorter, J.A., 2007. Complement activation in experimental and human temporal lobe epilepsy. Neurobiol. Dis. 26, 497-511.

Baraban, S.C., Taylor, M.R., Castro, P.A., Baier, H., 2005. Pentylenetetrazole induced changes in zebrafish behavior, neural activity and c-fos expression. Neuroscience 131, 759-768.

Barbazuk, W.B., Korf, I., Kadavi, C., Heyen, J., Tate, S., Wun, E., Bedell, J.A., McPherson, J.D., Johnson, S.L., 2000. The syntenic relationship of the zebrafish and human genomes. Genome Res. 10, 1351-1358.

Başaran, N., Hincal, F., Kansu, E., Ciğer, A., 1994. Humoral and cellular immune parameters in untreated and phenytoin-or carbamazepine-treated epileptic patients. Int. J. Immunopharmacol. 16, 1071-1077.

Bauer, J., 1996. Seizure-inducing effects of antiepileptic drugs: a review. Acta Neurol. Scand. 94, 367-377.

Benjamini, Y., Hochberg, Y., 1995. Controlling the False Discovery Rate: A Practical and Powerful Approach to Multiple Testing. J. R. Stat. Soc. Ser. B (Methodol. ) 57, 289-300.

Berghmans, S., Hunt, J., Roach, A., Goldsmith, P., 2007. Zebrafish offer the potential for a primary screen to identify a wide variety of potential anticonvulsants. Epilepsy Res. 75, 18-28.

Black, J.A., Waxman, S.G., 2008. Phenytoin protects central axons in experimental autoimmune encephalomyelitis. J. Neurol. Sci. 274, 57-63.

Black, J.A., Liu, S., Carrithers, M., Carrithers, L.M., Waxman, S.G., 2007. Exacerbation of experimental autoimmune encephalomyelitis after withdrawal of phenytoin and carbamazepine. Ann. Neurol. 62, 21-33.

Brodie, M.J., Covanis, A., Gil-Nagel, A., Lerche, H., Perucca, E., Sills, G.J., White, H.S., 2011. Antiepileptic drug therapy: does mechanism of action matter? Epilepsy Behav. 21, 331-341.

Chakraborty, C., Hsu, C.H., Wen, Z.H., Lin, C.S., Agoramoorthy, G., 2009. Zebrafish: a complete animal model for in vivo drug discovery and development. Curr. Drug Metab. 10, 116-124.

Chen, J.Q., Szodoray, P., Zeher, M., 2016. Toll-Like Receptor Pathways in Autoimmune Diseases. Clin. Rev. Allergy Immunol. 50, 1-17.

Christou, M., Kavaliauskis, A., Ropstad, E., Fraser, T.W.K., 2020. DMSO effects larval zebrafish (Danio rerio) behavior, with additive and interaction effects when combined with positive controls. Sci. Total Environ. 709, 134490.

Conolly, R.B., Lutz, W.K., 2004. Nonmonotonic dose-response relationships: mechanistic basis, kinetic modeling, and implications for risk assessment. Toxicol. Sci. 77, 151-157. 
Contin, M., Mohamed, S., Candela, C., Albani, F., Riva, R., Baruzzi, A., 2010. Simultaneous HPLC-UV analysis of rufinamide, zonisamide, lamotrigine, oxcarbazepine monohydroxy derivative and felbamate in deproteinized plasma of patients with epilepsy. J. Chromatogr. B Anal. Technol. Biomed. Life Sci. 878, $461-465$.

Coulthard, L.G., Woodruff, T.M., 2015. Is the Complement Activation Product C3a a Proinflammatory Molecule? Re-evaluating the Evidence and the Myth. J. Immunol. 194, 3542-3548.

Craner, M.J., Damarjian, T.G., Liu, S., Hains, B.C., Lo, A.C., Black, J.A., Newcombe, J., Cuzner, M.L., Waxman, S.G., 2005. Sodium channels contribute to microglia/ macrophage activation and function in EAE and MS. Glia 49, 220-229.

De Simoni, M.G., Perego, C., Ravizza, T., Moneta, D., Conti, M., Marchesi, F., De Luigi, A., Garattini, S., Vezzani, A., 2000. Inflammatory cytokines and related genes are induced in the rat hippocampus by limbic status epilepticus. Eur. J. Neurosci. 12, 2623-2633.

Dooley, K., Zon, L.I., 2000. Zebrafish: a model system for the study of human disease. Curr. Opin. Genet Dev. 10, 252-256.

Geijtenbeek, T.B., Gringhuis, S.I., 2009. Signalling through C-type lectin receptors: shaping immune responses. Nat. Rev. Immunol. 9, 465-479.

Gerlai, R., 2003. Zebra fish: an uncharted behavior genetic model. Behav. Genet 33, 461-468.

Godhwani, N., Bahna, S.L., 2016. Antiepilepsy drugs and the immune system. Ann. Allergy Asthma Immunol. Off. Publ. Am. Coll. 117, 634-640.

Griffin, A., Krasniak, C., Baraban, S.C., 2016. Advancing epilepsy treatment through personalized genetic zebrafish models. Prog. Brain Res. 226, 195-207.

Guerrini, R., Dravet, C., Genton, P., Belmonte, A., Kaminska, A., Dulac, O., 1998. Lamotrigine and Seizure Aggravation in Severe Myoclonic Epilepsy. Epilepsia 39, 508-512.

Himmerich, H., Bartsch, S., Hamer, H., Mergl, R., Schönherr, J., Petersein, C., Munzer, A., Kirkby, K.C., Bauer, K., Sack, U., 2013. Impact of mood stabilizers and antiepileptic drugs on cytokine production in-vitro. J. Psychiatr. Res. 47, 1751-1759.

Hortopan, G.A., Dinday, M.T., Baraban, S.C., 2010. Zebrafish as a model for studying genetic aspects of epilepsy. Dis. Model Mech. 3, 144-148.

Hossain, M.M., Sonsalla, P.K., Richardson, J.R., 2013. Coordinated role of voltage-gated sodium channels and the $\mathrm{Na}+/ \mathrm{H}+$ exchanger in sustaining microglial activation during inflammation. Toxicol. Appl. Pharmacol. 273, 355-364.

Howe, K., Clark, M.D., Torroja, C.F., Torrance, J., Berthelot, C., Muffato, M., Collins, J.E., Humphray, S., McLaren, K., Matthews, L., McLaren, S., Sealy, I., Caccamo, M., Churcher, C., Scott, C., Barrett, J.C., Koch, R., Rauch, G.J., White, S., Chow, W., Kilian, B., Quintais, L.T., Guerra-Assuncao, J.A., Zhou, Y., Gu, Y., Yen, J., Vogel, J.H., Eyre, T., Redmond, S., Banerjee, R., Chi, J., Fu, B., Langley, E., Maguire, S.F., Laird, G.K., Lloyd, D., Kenyon, E., Donaldson, S., Sehra, H., Almeida-King, J., Loveland, J., Trevanion, S., Jones, M., Quail, M., Willey, D., Hunt, A., Burton, J., Sims, S., McLay, K., Plumb, B., Davis, J., Clee, C., Oliver, K., Clark, R., Riddle, C., Elliot, D., Threadgold, G., Harden, G., Ware, D., Begum, S., Mortimore, B., Kerry, G., Heath, P., Phillimore, B., Tracey, A., Corby, N., Dunn, M., Johnson, C., Wood, J., Clark, S., Pelan, S., Griffiths, G., Smith, M., Glithero, R., Howden, P., Barker, N., Lloyd, C., Stevens, C., Harley, J., Holt, K., Panagiotidis, G., Lovell, J., Beasley, H., Henderson, C., Gordon, D., Auger, K., Wright, D., Collins, J., Raisen, C., Dyer, L., Leung, K., Robertson, L., Ambridge, K., Leongamornlert, D., McGuire, S., Gilderthorp, R., Griffiths, C., Manthravadi, D., Nichol, S., Barker, G., Whitehead, S., Kay, M., Brown, J., Murnane, C., Gray, E., Humphries, M., Sycamore, N., Barker, D., Saunders, D., Wallis, J., Babbage, A., Hammond, S., Mashreghi-Mohammadi, M., Barr, L., Martin, S., Wray, P., Ellington, A., Matthews, N., Ellwood, M., Woodmansey, R., Clark, G., Cooper, J., Tromans, A., Grafham, D., Skuce, C., Pandian, R., Andrews, R., Harrison, E., Kimberley, A., Garnett, J., Fosker, N., Hall, R., Garner, P., Kelly, D., Bird, C., Palmer, S., Gehring, I., Berger, A., Dooley, C. M., Ersan-Urun, Z., Eser, C., Geiger, H., Geisler, M., Karotki, L., Kirn, A., Konantz, J., Konantz, M., Oberlander, M., Rudolph-Geiger, S., Teucke, M., Lanz, C., Raddatz, G., Osoegawa, K., Zhu, B., Rapp, A., Widaa, S., Langford, C., Yang, F., Schuster, S.C., Carter, N.P., Harrow, J., Ning, Z., Herrero, J., Searle, S.M., Enright, A., Geisler, R., Plasterk, R.H., Lee, C., Westerfield, M., de Jong, P.J., Zon, L.I., Postlethwait, J.H., Nusslein-Volhard, C., Hubbard, T.J., Roest Crollius, H., Rogers, J., Stemple, D.L., 2013. The zebrafish reference genome sequence and its relationship to the human genome. Nature 496, 498-503.

Huang, D.W., Sherman, B.T., Tan, Q., Collins, J.R., Alvord, W.G., Roayaei, J., Stephens, R., Baseler, M.W., Lane, H.C., Lempicki, R.A., 2007. The DAVID Gene Functional Classification Tool: a novel biological module-centric algorithm to functionally analyze large gene lists. Genome Biol. 8, R183.

Huang da, W., Sherman, B.T., Lempicki, R.A., 2009. Systematic and integrative analysis of large gene lists using DAVID bioinformatics resources. Nat. Protoc. 4, 44-57.

Iori, V., Iyer, A.M., Ravizza, T., Beltrame, L., Paracchini, L., Marchini, S., Cerovic, M., Hill, C., Ferrari, M., Zucchetti, M., Molteni, M., Rossetti, C., Brambilla, R., Steve White, H., D'Incalci, M., Aronica, E., Vezzani, A., 2017. Blockade of the IL-1R1/TLR4 pathway mediates disease-modification therapeutic effects in a model of acquired epilepsy. Neurobiol. Dis. 99, 12-23.

Kim, D., Langmead, B., Salzberg, S.L., 2015. HISAT: a fast spliced aligner with low memory requirements. Nat. Methods 12, 357-360.

Kim, T., Kulick, C.G., Kortepeter, C.M., Brinker, A., Waldron, P., 2019. Hemophagocytic lymphohistiocytosis associated with the use of lamotrigine. Neurology 92, e2401-e2405.

Kohn, M.C., Melnick, R.L., 2002. Biochemical origins of the non-monotonic receptormediated dose-response. J. Mol. Endocrinol. 29, 113-123.

Lee, S.H., Kang, J.W., Lin, T., Lee, J.E., Jin, D.I., 2013. Teratogenic potential of antiepileptic drugs in the zebrafish model. Biomed. Res. Int. 2013, 726478.
Liao, Y., Smyth, G.K., Shi, W., 2014. featureCounts: an efficient general purpose program for assigning sequence reads to genomic features. Bioinformatics 30, 923-930.

Löscher, W., 2002. Animal models of epilepsy for the development of antiepileptogenic and disease-modifying drugs. A comparison of the pharmacology of kindling and post-status epilepticus models of temporal lobe epilepsy. Epilepsy Res. 50, 105-123.

Love, M.I., Huber, W., Anders, S., 2014. Moderated estimation of fold change and dispersion for RNA-seq data with DESeq2. Genome Biol. 15, 550.

Mathern, D.R., Heeger, P.S., 2015. Molecules Great and Small: The Complement System. Clin. J. Am. Soc. Nephrol. 10, 1636-1650.

Oby, E., Janigro, D., 2006. The blood-brain barrier and epilepsy. Epilepsia 47, 1761-1774.

Orellana-Paucar, A.M., Serruys, A.S., Afrikanova, T., Maes, J., De Borggraeve, W., Alen, J., León-Tamariz, F., Wilches-Arizábala, I.M., Crawford, A.D., de Witte, P.A. Esguerra, C.V., 2012. Anticonvulsant activity of bisabolene sesquiterpenoids of Curcuma longa in zebrafish and mouse seizure models. Epilepsy Behav. 24, 14-22.

Parsons, A., Lange, A., Hutchinson, T.H., Miyagawa, S., Iguchi, T., Kudoh, T., Tyler, C.R., 2019. Molecular mechanisms and tissue targets of brominated flame retardants, BDE-47 and TBBPA, in embryo-larval life stages of zebrafish (Danio rerio). Aquat. Toxicol. (Amst. Neth.) 209, 99-112.

Pernhorst, K., Herms, S., Hoffmann, P., Cichon, S., Schulz, H., Sander, T., Schoch, S., Becker, A.J., Grote, A., 2013. TLR4, ATF-3 and IL8 inflammation mediator expression correlates with seizure frequency in human epileptic brain tissue. Seizure $22,675-678$.

Ravizza, T., Gagliardi, B., Noe, F., Boer, K., Aronica, E., Vezzani, A., 2008. Innate and adaptive immunity during epileptogenesis and spontaneous seizures: evidence from experimental models and human temporal lobe epilepsy. Neurobiol. Dis. 29, $142-160$.

Rihel, J., Prober, D.A., Arvanites, A., Lam, K., Zimmerman, S., Jang, S., Haggarty, S.J., Kokel, D., Rubin, L.L., Peterson, R.T., Schier, A.F., 2010. Zebrafish behavioral profiling links drugs to biological targets and rest/wake regulation. Science 327, 348-351.

Roussel, B.D., Lomas, D.A., Crowther, D.C., 2016. Progressive myoclonus epilepsy associated with neuroserpin inclusion bodies (neuroserpinosis). Epileptic Disord. Int. Epilepsy J. Videotape 18, 103-110.

Schartz, N.D., Wyatt-Johnson, S.K., Price, L.R., Colin, S.A., Brewster, A.L., 2018. Status epilepticus triggers long-lasting activation of complement C1q-C3 signaling in the hippocampus that correlates with seizure frequency in experimental epilepsy. Neurobiol. Dis. 109, 163-173.

Smith, M., Wilcox, K.S., White, H.S., 2007. Discovery of antiepileptic drugs. Neurotherapeutics 4, 12-17.

Spina, E., Perugi, G., 2004. Antiepileptic drugs: indications other than epilepsy. Epileptic Disord. Int. Epilepsy J. Videotape 6, 57-75.

Stewart, A.M., Kalueff, A.V., 2012. The developing utility of zebrafish models for cognitive enhancers research. Curr. Neuropharmacol. 10, 263-271.

Strainic, M.G., Liu, J., Huang, D., An, F., Lalli, P.N., Muqim, N., Shapiro, V.S., Dubyak, G. R., Heeger, P.S., Medof, M.E., 2008. Locally produced complement fragments C5a and C3a provide both costimulatory and survival signals to naive CD4+ T cells. Immunity 28, 425-435.

Suzuki, Y., Hashimoto, K., Hoshi, K., Ito, H., Kariya, Y., Miyazaki, K., Sato, M., Kawasaki, Y., Yoshida, M., Honda, T., Hashimoto, Y., Hosoya, M., 2019. Ratio of Alpha 2-Macroglobulin Levels in Cerebrospinal Fluid and Serum: An Expression of Neuroinflammation in Acute Disseminated Encephalomyelitis. Pedia Neurol. 98, 61-67.

Svalheim, S., Mushtaq, U., Mochol, M., Luef, G., Rauchenzauner, M., Froland, S.S., Tauboll, E., 2013. Reduced immunoglobulin levels in epilepsy patients treated with levetiracetam, lamotrigine, or carbamazepine. Acta Neurol. Scand. Suppl. 11-15.

Uludag, I.F., Duksal, T., Tiftikcioglu, B.I., Zorlu, Y., Ozkaya, F., Kirkali, G., 2015. IL-1ß, IL-6 and IL1Ra levels in temporal lobe epilepsy. Seizure 26, 22-25.

van Vliet, E.A., Aronica, E., Vezzani, A., Ravizza, T., 2018. Review: Neuroinflammatory pathways as treatment targets and biomarker candidates in epilepsy: emerging evidence from preclinical and clinical studies. Neuropathol. Appl. Neurobiol. 44, 91-111.

Varma, V.R., Varma, S., An, Y., Hohman, T.J., Seddighi, S., Casanova, R., Beri, A., Dammer, E.B., Seyfried, N.T., Pletnikova, O., Moghekar, A., Wilson, M.R., Lah, J.J., O’Brien, R.J., Levey, A.I., Troncoso, J.C., Albert, M.S., Thambisetty, M., 2017. Predictors of Cognitive Decline Among Normal, I., the Alzheimer's Disease Neuroimaging Initiative, s., 2017. Alpha-2 macroglobulin in Alzheimer's disease: a marker of neuronal injury through the RCAN1 pathway. Mol. Psychiatry 22, 13-23.

Vezzani, A., Granata, T., 2005. Brain inflammation in epilepsy: experimental and clinical evidence. Epilepsia 46, 1724-1743.

Vezzani, A., Conti, M., De Luigi, A., Ravizza, T., Moneta, D., Marchesi, F., De Simoni, M. G., 1999. Interleukin-1beta immunoreactivity and microglia are enhanced in the rat hippocampus by focal kainate application: functional evidence for enhancement of electrographic seizures. J. Neurosci. 19, 5054-5065.

Vezzani, A., Moneta, D., Conti, M., Richichi, C., Ravizza, T., De Luigi, A., De Simoni, M. G., Sperk, G., Andell-Jonsson, S., Lundkvist, J., Iverfeldt, K., Bartfai, T., 2000. Powerful anticonvulsant action of IL-1 receptor antagonist on intracerebral injection and astrocytic overexpression in mice. Proc. Natl. Acad. Sci. USA 97, 11534-11539.

Vezzani, A., Balosso, S., Ravizza, T., 2019. Neuroinflammatory pathways as treatment targets and biomarkers in epilepsy. Nat. Rev. Neurol. 15, 459-472.

Yamamoto, T., Uchiyama, T., Takahashi, H., Himuro, K., Kanai, K., Kuwabara, S., 2010. B cell aplasia and hypogammaglobulinemia after carbamazepine treatment. Intern. Med. (Tokyo Jpn.) 49, 707-708.

Yu, G., Wang, L.-G., Han, Y., He, Q.-Y., 2012. clusterProfiler: an R package for comparing biological themes among gene clusters. OMICS 16, 284-287. 